\title{
Microscopic calculation of symmetry projected nuclear level densities
}

\author{
K. Van Houcke, ${ }^{1}$ S. M. A. Rombouts, ${ }^{2}$ K. Heyde, ${ }^{1}$ and Y. Alhassid ${ }^{3}$ \\ ${ }^{1}$ Universiteit Gent-UGent, Vakgroep Subatomaire en Stralingsfysica, Proeftuinstraat 86, B-9000 Gent, Belgium \\ ${ }^{2}$ Department of Physics and Astronomy, Aarhus University DK-8000, Aarhus C, Denmark \\ ${ }^{3}$ Center for Theoretical Physics, Sloane Physics Laboratory, Yale University New Haven, Connecticut 06520, USA \\ (Received 9 February 2007; revised manuscript received 3 September 2008; published 3 February 2009)
}

\begin{abstract}
We present a quantum Monte Carlo method with exact projection on parity and angular momentum that is free of a sign problem for seniority-conserving nuclear interactions. This technique allows the microscopic calculation of angular momentum and parity-projected nuclear level densities. We present results for the ${ }^{55} \mathrm{Fe},{ }^{56} \mathrm{Fe}$, and ${ }^{57} \mathrm{Fe}$ isotopes. Signatures of the pairing phase transition are observed in the angular momentum distribution of the nuclear level density.
\end{abstract}

DOI: 10.1103/PhysRevC.79.024302

PACS number(s): 21.10.Ma, 21.60.Cs, 21.60.Ka, 27.40.+z

The level density is a fundamental property of a many-body system as all thermodynamical quantities can be derived from it. In nuclear physics, level densities are important because they are required for estimating nuclear reaction rates. The assumption of a free Fermi gas leads to the well-known Bethe formula for the level density [1,2]. A phenomenological way of incorporating pairing correlations and shell effects is to back-shift the excitation energy $E_{x}$ by an amount $\Delta$, resulting in the backshifted Bethe formula.

The calculation of statistical nuclear reaction rates also requires knowledge of the angular momentum distribution of the nuclear level density. An empirical formula for the angular momentum distribution of the level density at a fixed excitation energy $E_{x}$ assumes uncorrelated and randomly coupled single-particle spins and is given by $[3,4]$

$$
\rho_{J}\left(E_{x}\right)=\rho\left(E_{x}\right) \frac{(2 J+1)}{2 \sqrt{2 \pi} \sigma^{3}} e^{-\frac{J(J+1)}{2 \sigma^{2}}} .
$$

Here $J$ is the total angular momentum and $\sigma$ is a spin-cutoff parameter that can be used as an energy dependent fitting parameter. The total level density $\rho\left(E_{x}\right)$ in Eq. (1) is often parametrized by a backshifted Bethe formula. The main disadvantage of this approach is that it requires a fit for each individual nucleus.

The microscopic calculation of the level density, and in particular its angular momentum distribution beyond the Fermi gas model and the spin-cutoff parametrization of Eq. (1), poses a complex problem. Recently, progress has been made both experimentally [5,6] and theoretically [7] in gaining more insight into the angular momentum distribution of level densities. The level density has been calculated microscopically using the shell model Monte Carlo (SMMC) method [8-13]. In the SMMC the effect of residual interactions between the nucleons is taken into account, although restricted to pairing plus multipole-multipole interactions that are free of the Monte Carlo sign problem. Angular momentum projection in the SMMC method was recently carried out for scalar observables by first projecting on the angular momentum component $J_{z}$ [7]. Angular momentum projection, however, introduces a new sign problem for $J \neq 0$ even for a good sign interaction.

In the present study, we discuss a quantum Monte Carlo (QMC) method to solve a general isovector $J=0$ pairing model and carry out an angular momentum projection for seniority-conserving models. The pairing model serves as a benchmark to identify signatures of pairing correlations in the nuclear level density. Our QMC method allows us to treat the very large model spaces that are needed for the calculation of level densities at higher excitation energies and, particularly, for the study of their parity and angular momentum dependence.

We start from a general isovector $J=0$ pairing model, here written in the quasi-spin formalism $[14,15]$

$$
\begin{aligned}
& \hat{H}=\sum_{j} 2 \varepsilon_{j} \hat{S}_{j}^{0}-\sum_{j j^{\prime}} g_{j j^{\prime}} \hat{S}_{j}^{+} \hat{S}_{j^{\prime}}^{-}, \\
& \hat{S}_{j}^{0}=\frac{1}{2} \sum_{m>0}\left(\hat{a}_{j m}^{\dagger} \hat{a}_{j m}+\hat{a}_{j \bar{m}}^{\dagger} \hat{a}_{j \bar{m}}-1\right), \\
& \hat{S}_{j}^{+}=\sum_{m>0} \hat{a}_{j m}^{\dagger} \hat{a}_{j \bar{m}}^{\dagger}, \quad \hat{S}_{j}^{-}=\sum_{m>0} \hat{a}_{j \bar{m}} \hat{a}_{j m},
\end{aligned}
$$

where the operator $\hat{a}_{j m}^{\dagger}$ creates a particle in the mean-field single-particle state $|j m\rangle$ with energy $\varepsilon_{j}$ and $|j \bar{m}\rangle$ is the timereversed conjugate state of $|j m\rangle$. The operators $\hat{S}_{j}^{0}, \hat{S}_{j}^{+}$, and $\hat{S}_{j}^{-}$ close an SU(2) algebra and are known as quasi-spin operators. The Hamiltonian (2) is exactly solvable for a constant pairing strength. The QMC method discussed here allows us to solve the general case when all terms in Eq. (2) are attractive, i.e., $g_{j j^{\prime}}>0$.

We formulate the pairing problem in the quasi-spin formalism to address the problem of the angular momentum projection. The quasi-spin projection $S_{j}^{0}$ determines the total number of particles $N_{j}=2 S_{j}^{0}+\Omega_{j}$ in level $j\left(\Omega_{j}=j+1 / 2\right.$ is the pair degeneracy of orbital $j$ ), while the quasi-spin quantum number $S_{j}$ determines the seniority quantum number $v_{j}=\Omega_{j}-2 S_{j}$, i.e., the number of unpaired particles in level $j$. Within this quasi-spin (or seniority) scheme, angular momentum remains a good quantum number. Given a set of quasi-spin quantum numbers $S_{j}$, one can determine the degeneracy for a given value of the total angular momentum $J$. Formally, the problem is equivalent to a chain of spins. The pairing interaction can flip spins such that the total "magnetization" $\sum_{j} S_{j}^{0}$ remains constant (particle number 
conservation). In addition, each quasi-spin quantum number $S_{j}$ can take values between 0 and $\Omega_{j} / 2$.

There exist a number of very efficient QMC approaches to simulate spin chains [16]. However, in our quasi-spin model it is necessary to implement a method that will keep the value of the magnetization $\sum_{j} S_{j}^{0}$ fixed. Recently, we developed a nonlocal loop update QMC scheme that is capable of sampling spin models at constant magnetization. In our case this means sampling the canonical ensemble $[17,18]$. Assuming the Hamiltonian $\hat{H}=\hat{H}_{0}-\hat{V}$ consists of two noncommuting parts $\hat{H}_{0}$ and $\hat{V}$, our scheme is based on a perturbative expansion of the partition function at inverse temperature $\beta$,

$$
\begin{aligned}
\operatorname{Tr}\left(e^{-\beta \hat{H}}\right)= & \sum_{m=0}^{\infty} \int_{0}^{\beta} d \tau_{m} \int_{0}^{\tau_{m}} d \tau_{m-1} \cdots \int_{0}^{\tau_{2}} d \tau_{1} \\
& \times \operatorname{Tr}\left[\hat{V}\left(\tau_{1}\right) \hat{V}\left(\tau_{2}\right) \cdots \hat{V}\left(\tau_{m}\right) e^{-\beta \hat{H}_{0}}\right],
\end{aligned}
$$

with $\hat{V}(\tau)=\exp \left(-\tau \hat{H}_{0}\right) \hat{V} \exp \left(\tau \hat{H}_{0}\right)$. Here we choose

$\hat{H}_{0}=\sum_{j} 2 \varepsilon_{j} \hat{S}_{j}^{0}-\sum_{j} g_{j j} \hat{S}_{j}^{+} \hat{S}_{j}^{-} ; \quad \hat{V}=\sum_{j \neq j^{\prime}} g_{j j^{\prime}} \hat{S}_{j}^{+} \hat{S}_{j^{\prime}}^{-}$.

The basic idea of the QMC method is to insert a so-called worm operator $\hat{A}$ in the partition function, obtaining an extended partition function $\operatorname{Tr}\left(\hat{A} e^{-\beta \hat{H}}\right)$. By propagating this worm operator through imaginary time according to the rules explained in Refs. [17,18], one generates configurations that are distributed according to their weight in the canonical partition function $\operatorname{Tr}_{N}\left(e^{-\beta \hat{H}}\right)$ at fixed particle number $N$. Here, the worm operator is chosen to be $C+\sum_{i j} S_{i}^{+} S_{j}^{-}$, with $C$ a constant. Such a worm operator allows for the sampling of all configurations that correspond to a fixed set of quasi-spin quantum numbers $S_{j}$ without changing the value of $\sum_{j} S_{j}^{0}$. However, to be ergodic, the worm operator must also generate configurations with varying seniority quantum numbers, and therefore we add a worm operator that can change the values of the quasi-spins $S_{i}, S_{j}$ and their projections $S_{i}^{0}, S_{j}^{0}$ for two levels $i$ and $j$ such that $\left(S_{i}^{0}+S_{j}^{0}\right)$ remains the same (particle-number conservation). The complete quasi-spin phase space is now sampled by propagating a seniority-conserving worm operator and an additional seniority nonconserving worm operator. Additional details on the worm operator moves can be found in Refs. [17,18].

The moves of the worm operator can be constrained in such a way that the angular momentum $J$ of the configuration does not change. Choosing an initial configuration with a welldefined angular momentum quantum number $J$ enables us to calculate thermal averages for that fixed value of $J$. Parity is also a good quantum number in the quasi-spin basis, so that parity projection can be carried out in a similar way.

We have used the QMC method outlined above to study the angular momentum and parity distribution of nuclear level densities in the presence of pairing correlations. We focused on the iron isotopes ${ }^{55} \mathrm{Fe},{ }^{56} \mathrm{Fe}$, and ${ }^{57} \mathrm{Fe}$, within the complete $0 f+1 p+0 g_{9 / 2}$ model space. To study truncation effects, we also consider an extended model space $1 s+0 d+0 f+1 p+$ $0 g_{9 / 2}+2 s+1 d$. The inverse temperature $\beta$ ranges from 0 to $2.5 \mathrm{MeV}^{-1}$. For the mean-field one-body potential, we used a
Woods-Saxon potential with the parametrization of Ref. [19]. The single-particle energies $\varepsilon_{j}$ were obtained by diagonalizing this potential in a harmonic oscillator basis. For simplicity, we used a constant pairing strength $g_{j j^{\prime}}=g$ (although our QMC method is not restricted to this case), determined to reproduce the experimental gap parameter $\Delta=-1 / 2[\mathcal{B}(N-1, Z)-$ $2 \mathcal{B}(N, Z)+\mathcal{B}(N+1, Z)]$ for $Z$ protons and $N$ neutrons ( $\mathcal{B}$ denotes the binding energy) [2]. For the larger valence space, the value of pairing strength $g$ is renormalized in such a way that the pairing gap $\Delta$ remained fixed. Based on the temperatures for which the heat capacity is maximal, we estimate our calculations to be free of truncation effects for excitation energies up to $E_{x} \lesssim 20 \mathrm{MeV}$ and $E_{x} \lesssim 50 \mathrm{MeV}$ for the $p f+g_{9 / 2}$ and $s d+p f+g_{9 / 2}+s d$ valence spaces, respectively. Total and angular momentum/parity-projected level densities are calculated as usual in the saddle-point approximation.

Figure 1 shows the angular momentum distribution of the projected level density $\rho_{J}$ at four different excitation energies $E_{x}$ [we do not include the magnetic degeneracy in $\rho_{J}$, i.e., $\left.\rho\left(E_{x}\right)=\sum_{J}(2 J+1) \rho_{J}\left(E_{x}\right)\right]$. The solid squares are the QMC results (the statistical errors are much smaller than the size of the squares), while the solid lines are the spin-cutoff model Eq. (1) fitted to the QMC data with $\sigma^{2}$ as a fit parameter. For all three iron isotopes, the angular momentum distribution becomes broader with increasing excitation energy. When this energy is sufficiently high $\left(E_{x} \geqslant 10 \mathrm{MeV}\right)$, the QMC data are well described by the spin-cutoff model. For excitation energies $\leqslant 10 \mathrm{MeV}$, some deviations are observed. In particular,

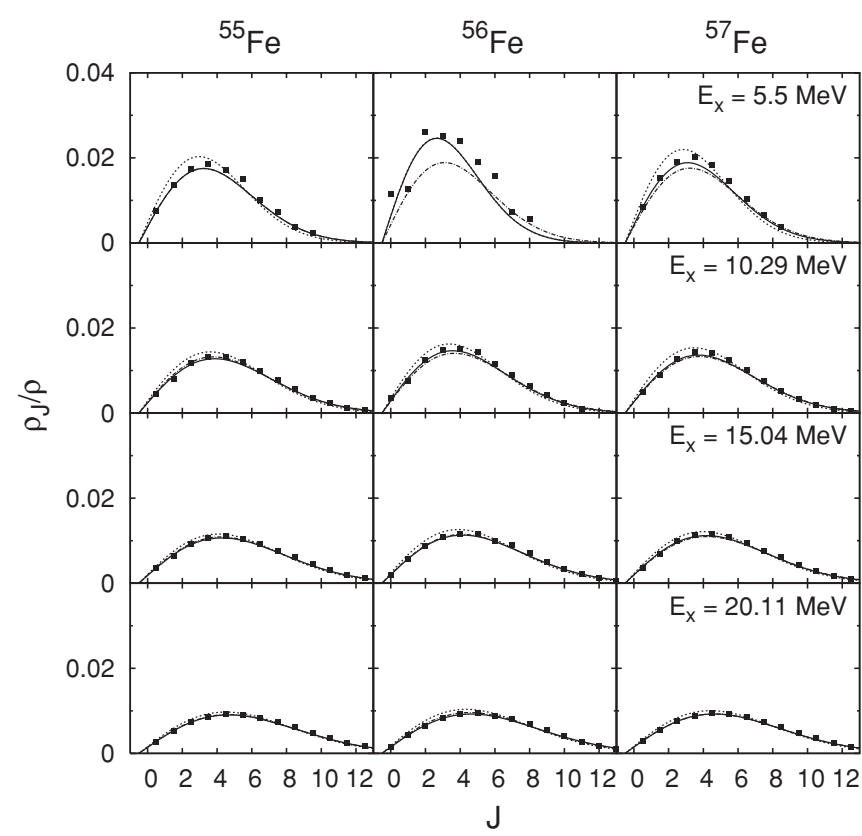

FIG. 1. The angular momentum distribution of the level density at a given excitation energy $E_{x}$ for the iron isotopes ${ }^{55} \mathrm{Fe},{ }^{56} \mathrm{Fe}$, and ${ }^{57} \mathrm{Fe}$. Solid squares are the Monte Carlo results in the $p f+g_{9 / 2}$ valence space. The solid lines are fits to the spin-cutoff model Eq. (1). Also shown are distributions given by the spin-cutoff model with $\sigma^{2}$ calculated from Eq. (7) using the rigid body moment of inertia (dotted-dashed lines) and from Eq. (6) (dotted lines). 


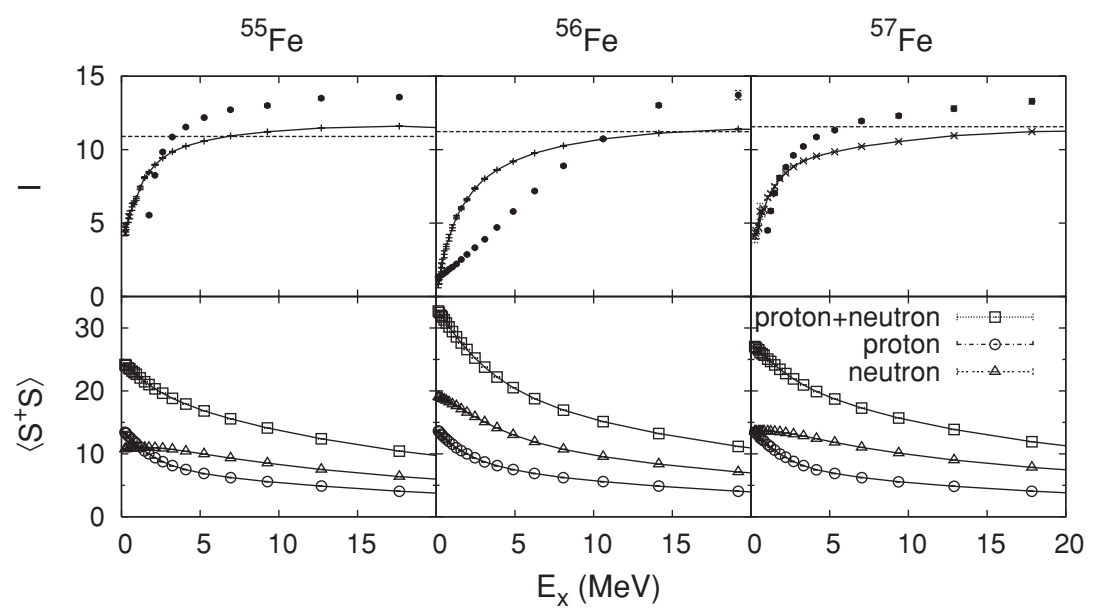

FIG. 2. (Top panels) The moment of inertia $I$ (in units $\hbar^{2}$ ) for ${ }^{55} \mathrm{Fe}$ (left), ${ }^{56} \mathrm{Fe}$ (middle), and ${ }^{57} \mathrm{Fe}$ (right) versus excitation energy $E_{x}$. The solid lines are moments of inertia calculated from Eqs. (7) and (6), and the solid circles are from Eq. (7) with $\sigma^{2}$ determined by $\rho_{0} / \rho$ (in the even-even case) and $\rho_{1 / 2} / \rho$ (in the odd-even case). The horizontal dashed lines describe the rigid-body moments of inertia. (Bottom panels) The pair correlation energy $\left\langle S^{\dagger} S\right\rangle$. a staggering effect in $J$ is found for the even-even ${ }^{56} \mathrm{Fe}$ isotope but not in the odd-even iron isotopes. The strong suppression of the $J=1$ level density $\left(\rho_{1} \approx \rho_{0}\right)$, which is much smaller than the $J=2$ level density, is a signature of the pairing correlations. For higher angular momenta, there is only a very small staggering in the angular momentum dependence. This can be understood from the fact that the pairing interaction scatters only $J=0$ nucleon pairs.

In the spin-cutoff model (1), the parameter $\sigma$ is given by

$$
\sigma^{2}=\frac{1}{3}\left\langle\hat{J}^{2}\right\rangle
$$

with $\langle\ldots\rangle$ denoting a thermal average at temperature $T$ [20]. It is then common to define a semiclassical effective moment of inertia from

$$
I=\frac{\hbar^{2}}{T} \sigma^{2} .
$$

The spin-cutoff parameter is often determined by substituting the rigid-body moment of inertia, $I=2 m A\left(r_{0} A^{1 / 3}\right)^{2} / 5$ in Eq. (7). (Here, $r_{0}$ is the nuclear radius parameter, $A$ is the mass number, and $m$ is the nucleon mass). For $E_{x} \geqslant 10 \mathrm{MeV}$, the spin-cutoff model (1) with $\sigma^{2}$ calculated from the rigid-body moment of inertia (dotted-dashed lines in Fig. 1) essentially coincides with the distribution fitted to the Monte Carlo data (solid lines in Fig. 1). However, below $E_{x} \sim 10 \mathrm{MeV}$ and for ${ }^{56} \mathrm{Fe}$, the rigid-body moment of inertia predicts a broader angular momentum distribution than the one described by the fit to the QMC results. This indicates a reduction of the effective moment of inertia because of pairing correlations and can be regarded as a signature of nuclear superfluidity. We also determined $\sigma^{2}$ from Eq. (6) with $\left\langle\hat{J}^{2}\right\rangle$ calculated directly in the QMC simulations. The angular momentum distributions using these spin-cutoff parameters are shown by the dotted lines in Fig. 1. We observe that these distributions are slightly more peaked than the fitted distributions (solid lines), especially at low excitation energies $\left(E_{x} \leqslant 10 \mathrm{MeV}\right)$. For ${ }^{56} \mathrm{Fe}$ at $E_{x}=$ $5.5 \mathrm{MeV}$, this curve coincides with the fit.

The top panels of Fig. 2 show the effective moment of inertia as a function of excitation energy. At high excitation energies, the moment of inertia calculated from $\left\langle\hat{J}^{2}\right\rangle$ (solid lines) is very close to its rigid-body value, the latter indicated by the horizontal dashed lines. We also calculated the moment of inertia using the $\sigma^{2}$ obtained by fitting the QMC angular momentum distribution. At low excitation energy, however, we found that the error bars in this $\sigma^{2}$ increase, making the fit less meaningful. Therefore, we calculated the spin-cutoff parameter $\sigma^{2}$ directly from the ratio $\rho_{0(1 / 2)} / \rho$ in the even (odd) case. The corresponding moments of inertia are also shown in the top panels of Fig. 2 (solid circles). Although different methods for calculating $\sigma^{2}$ lead to different values of $I$, these differences do not lead to significant differences in the angular momentum distribution shown in Fig. 1. In general, we find that the moment of inertia of ${ }^{56} \mathrm{Fe}$ is strongly suppressed compared to the moments of inertia of ${ }^{55} \mathrm{Fe}$ and ${ }^{57} \mathrm{Fe}$.

The bottom panels of Fig. 2 show the pair correlation energy $\left\langle S^{+} S^{-}\right\rangle=\left\langle\sum_{j, j^{\prime}} S_{j}^{+} S_{j^{\prime}}^{-}\right\rangle$as a function of excitation energy. It is seen that the pair correlation energy is strongly suppressed with increasing excitation energy. The suppressed neutron correlation energy of ${ }^{55} \mathrm{Fe}$ and ${ }^{57} \mathrm{Fe}$ shows a slight increase at low excitations with increased excitation energy (or temperature). This reflects a blocking effect of the unpaired neutron at low temperatures.

In Fig. 3 we show the level density projected on parity and the four lowest angular momentum values as a function of

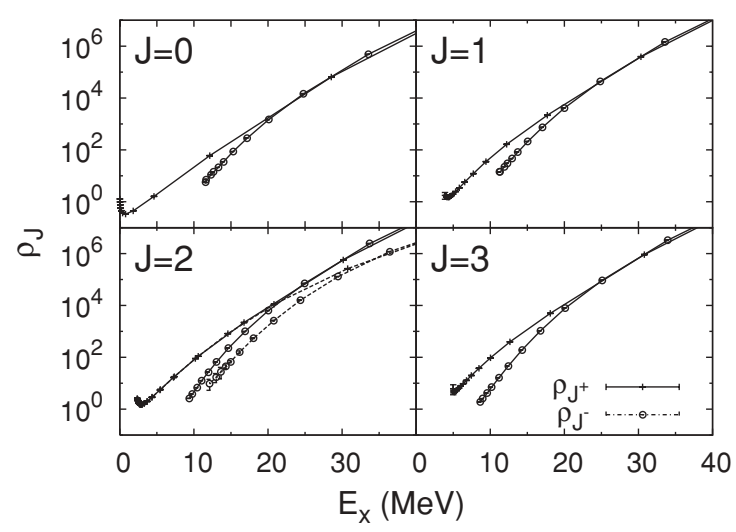

FIG. 3. The angular momentum and parity-projected level densities of ${ }^{56} \mathrm{Fe}$ for the four lowest angular momentum values. A strong parity dependence is found for excitation energies up to $\sim 20 \mathrm{MeV}$. The valence model space is $s d+p f+g_{9 / 2}+s d$. For $J=2$, we also show results for the smaller $p f+g_{9 / 2}$ valence space (dashed lines). 


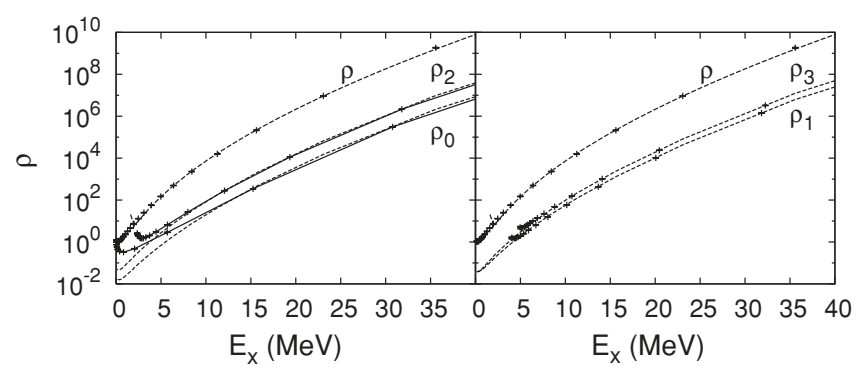

FIG. 4. The total QMC level density for ${ }^{56} \mathrm{Fe}$, well described by the backshifted Bethe formula (dotted-dashed line), together with the projected densities $J=0, J=2$ (left panel) and $J=1, J=3$ (right panel). The solid lines connect the $J$-projected Monte Carlo results, while the dashed lines show the projected level density from the spin-cutoff model Eq. (1) using rigid-body values of $\sigma^{2}$.

excitation energy using the $s d+f p g+s d$ valence space. For energies below $\sim 20 \mathrm{MeV}$, the angular momentum projected level densities show a strong parity dependence. Recently, $J=$ 2 parity-projected level densities were determined experimentally in ${ }^{58} \mathrm{Ni}$ at intermediate excitation energies [6]. However, no parity dependence was observed in the experiments. Our calculations show that the angular momentum projected level density displays a strong parity dependence. For $J=2$, we also show in Fig. 3 the level density within the smaller $p f+g_{9 / 2}$ valence space (dashed lines). Below $\sim 20 \mathrm{MeV}$, the even-parity level density is in good agreement with the results found in the $s d+f p g+s d$ model space. However, the inclusion of the $s d$ shells below and above the $p f+g_{9 / 2}$ shell enhances significantly the odd-parity level density at low excitation energies. We found a similar effect for the total odd-parity level density. This results from the increased fraction of single-particle levels with positive parity and shows the necessity of using large model spaces.

In the left (right) panel of Fig. 4 we show the total QMC level density, as well as the angular momentum projected level densities $J=0(J=1)$ and $J=2(J=3)$, for ${ }^{56} \mathrm{Fe}$. The total QMC level density is well described by the backshifted Bethe formula (fitted for $4.5 \mathrm{MeV}<E_{x}<40 \mathrm{MeV}$ ) with a singleparticle level density parameter $a=5.741 \pm 0.034 \mathrm{MeV}^{-1}$ and a backshift $\Delta=1.591 \pm 0.057 \mathrm{MeV}$ (see dotted-dashed line in Fig. 4). These are similar to the SMMC level density parameters $a=5.780 \pm 0.055 \mathrm{MeV}^{-1}$ and $\Delta=1.560 \pm$ $0.161 \mathrm{MeV}$ of Ref. [9], which were found to be in good agreement with the experimental level density. The dashed lines of Fig. 4 are the angular momentum projected level densities, calculated from the total level density through Eq. (1) with a rigid-body value of $\sigma^{2}$. At high excitation energy, these densities coincide with the QMC densities. However, for excitation energies below $\sim 10 \mathrm{MeV}$, the $J=0$ QMC level density deviates from the level density predicted by the spin-cutoff model with a rigid body $\sigma^{2}$. This can be interpreted as a signature of the pairing phase transition in the level density. This signature is also visible in the $J=2$ level density for energies below $\sim 8 \mathrm{MeV}$. For odd $J$ values, however, the QMC data are well described by the rigid-body model.

In conclusion, we have used a quantum Monte Carlo method to calculate angular momentum and parity-projected level densities for a nuclear pairing model. This method allows us to calculate level densities in the very large model spaces. We have found that pairing correlations affect the angular momentum distribution of the level density at low excitation energies, thereby revealing signatures of the pairing phase transition in nuclei.

\section{ACKNOWLEDGMENTS}

We thank N. Jachowicz, K. Langanke, and A. Richter for interesting suggestions and discussions. We acknowledge the financial support of the Fund for Scientific Research-Flanders (Belgium) and IUAP (P5/07 and P6/23) and of US DOE Grant DE-FG-0291-ER-40608.
[1] H. A. Bethe, Phys. Rev. 50, 332 (1936); Rev. Mod. Phys. 9, 69 (1937).

[2] A. Bohr and B. R. Mottelson, Nuclear Structure (Benjamin, New York, 1969), Vol. I.

[3] C. Bloch, Phys. Rev. 93, 1094 (1954).

[4] T. Ericson, Adv. Phys. 9, 425 (1960).

[5] Y. Kalmykov et al., Phys. Rev. Lett. 96, 012502 (2006).

[6] Y. Kalmykov, C. Ozen, K. Langanke, G. Martinez-Pinedo, P. von Neumann-Cosel, and A. Richter, Phys. Rev. Lett. 99, 202502 (2007).

[7] Y. Alhassid, S. Liu, and H. Nakada, Phys. Rev. Lett. 99, 162504 (2007).

[8] S. E. Koonin, D. J. Dean, and K. Langanke, Phys. Rep. 278, 1 (1997).

[9] H. Nakada and Y. Alhassid, Phys. Rev. Lett. 79, 2939 (1997).

[10] W. E. Ormand, Phys. Rev. C 56, R1678 (1997).
[11] S. Rombouts, K. Heyde, and N. Jachowicz, Phys. Rev. C 58, 3295 (1998).

[12] K. Langanke, Phys. Lett. B438, 235 (1998).

[13] Y. Alhassid, G. F. Bertsch, S. Liu, and H. Nakada, Phys. Rev. Lett. 84, 4313 (2000).

[14] D. J. Dean and M. Hjorth-Jensen, Rev. Mod. Phys. 75, 607 (2003).

[15] D. M. Brink and R. A. Broglia, Nuclear Superfluidity (Cambridge University Press, Cambridge, 2005).

[16] N. Kawashima and K. Harada, J. Phys. Soc. Jpn. 73, 1379 (2004).

[17] S. M. A. Rombouts, K. Van Houcke, and L. Pollet, Phys. Rev. Lett. 96, 180603 (2006).

[18] K. Van Houcke, S. M. A. Rombouts, and L. Pollet, Phys. Rev. E 73, 056703 (2006).

[19] C. M. Perey and F. G. Perey, Nucl. Data Tables 10, 539 (1972).

[20] Y. Alhassid, G. F. Bertsch, L. Fang, and S. Liu, Phys. Rev. C 72, 064326 (2005). 